\title{
Molekulare Stoffwechselsensorik bei durch Adipositas induzierter Entzündung*
}

\author{
Elisabeth Eisenstecken ${ }^{a} \quad$ Johanna Maria Gostner ${ }^{b} \quad$ Dietmar Fuchs $^{c} \quad$ Florian Überall $^{b}$ \\ ${ }^{a}$ Allgemein öffentliches Bezirkskrankenhaus Kufstein, \\ bSektion für Medizinische Biochemie, \\ 'Sektion für Biologische Chemie, Centrum für Chemie und Biowissenschaften, Medizinische Universität Innsbruck, Österreich
}

\section{Schlüssselwörter}

Adipozytokine $\cdot$ Fettgewebsentzündung $\cdot$ Metaflammation .

Nutrisensorik $\cdot$ Metabolische Sensorsysteme

\section{Zusammenfassung}

Metabolische Dysfunktionen und damit einhergehende chronische Entzündungsprozesse gefährden die Aufrechterhaltung der regulären zellulären Signalweiterleitung und verursachen so persistierende Störungen der Stoffwechsel- und Immunhomöodynamik. Diese Erkrankungen werden durch chronische Stressstimuli wie hyperkalorische Ernährung oder Fehlernährung ausgelöst und manifestieren sich vorwiegend als metabolische Stoffwechselstörungen, die mit einem systemischen Entzündungsstatus (Metaflammation) assoziiert sind. Die Anzahl der Begleit- und Folgeerkrankungen solcher metabolischimmunologischer Dysfunktionen nimmt rapide zu, sodass man beinahe von einem pandemieartigen Auftreten sprechen kann. Die adipöse Metaflammation ist durch eine massive Verschiebung der Energiebilanz in den beteiligten Zellsystemen und durch die Modulation wichtiger immunologischer Prozesse gekennzeichnet. Bei metabolischen Entgleisungen sind sowohl Adipozyten als auch Makrophagen bedeutende zelluläre Sensoren und zugleich Effektoren, was auch durch die enge genetische und funktionelle Verknüpfung der beiden Zelltypen erklärt werden kann. Auch das Zentrale Nervensystem ist stark in die Kontrolle der metabolischen Homöostase eingebunden. Kommt es ernährungsbedingt zu einer krankhaften Zunahme des weissen, viszeralen Fettgewebes, tritt ausgelöst durch eine Störung der Adipozytokinfreisetzung und die erhöhte Zirkulation freier Fettsäuren eine vorerst milde Entzündungsreaktion auf. Gelingt es innerhalb eines bestimmten Zeitraums nicht, die metabolische Störung zu revidieren, werden vermehrt Immunzellen ins Fettgewebe rekrutiert. Eine chronische Fettgewebs-

*Teile dieses Beitrags basieren auf der Diplomarbeit von E. Eisenstecken, Medizinische Universität Innsbruck, Österreich, und wurden von den Autoren entsprechend aktualisiert und erweitert. entzündung führt zur Entwicklung von Insulinresistenz und löst weitere pathophysiologische Mechanismen aus. Diese wirken sich systemisch aus und betreffen auch Gewebe in der Peripherie. Derartige Prozesse stellen ein neuartiges Entzündungsphänomen dar, da die Zellantwort nicht bakteriell oder viral, sondern durch persistierende metabolische Entgleisungen ausgelöst wird.

\section{Keywords}

Adipokine · Adipose tissue inflammation - Metaflammation .

Nutrisensing $\cdot$ Metabolic sensor systems

\section{Summary \\ Molecular Metabolic Sensors in Obesity-Induced Inflammation}

Metabolic dysfunctions and associated chronic inflammatory processes threaten regular cellular signal transduction and cause persistent disturbances of metabolic and immunological mediated hemodynamics. These disorders are provoked by chronic stress events such as hypercaloric nutrition or malnutrition and manifest themselves as metabolic disturbances accompanied by systemic inflammatory reactions (metaflammation). The number of resulting complications increases continuously and reaches almost pandemic proportions. An adipose metaflammation is characterized by the modulation of important immune processes as well as a severe shift in the energy balance of all cell systems involved. Both adipocytes and macrophages represent important sensors and effectors for metabolic disturbances, which also highlights their genetic and functional similarities. Additionally, the central nervous system is essential in the control of the metabolic hemodynamic. In case of a pathophysiological increase of the white, visceral adipose tissue, the abnormal secretion of adipokines and the circulation of high amounts of fatty acids provoke an initially mild and local inflammation. If the metabolic dysfunctions cannot be successfully corrected within a certain period of time, an augmented immune response is triggered by newly recruited immune cells. This chronic adipose tissue inflammation leads to the development of insulin

$\begin{array}{ll}\text { KARGER } & \begin{array}{l}\text { ○ 2012 S. Karger GmbH, Freiburg } \\ 1015-0684 / 12 / 0244-0221 \$ 38.00 / 0\end{array} \\ \begin{array}{l}\text { Fax }+497614520714 \\ \text { Information@Karger.de } \\ \text { www.karger.com }\end{array} & \begin{array}{l}\text { Accessible online at: } \\ \text { www.karger.com/szg }\end{array}\end{array}$

Ao. Univ. Prof. Mag . Dr. Florian Überall, PhD

Nutritional Biochemistry and Nutrigenomics, Division of Medical Biochemistry Centrum für Chemie und Biowissenschaften, Medizinische Universität Innsbruck Innrain 80-82, 6020 Innsbruck, Österreich

Tel. +43512 9003-70120, Fax -73130

florian.ueberall@i-med.ac.at 
resistance and other pathological mechanisms, which also systemically affect peripheral tissue. Such processes represent a particular inflammatory phenomenon as the cell response is not bacterial or viral but is triggered by persistent metabolic disturbances.

\section{Mots-clés}

Adipocytokine · Inflammation des tissus adipeux · Méta-inflammation . Nutrisensorique $\cdot$ Systèmes de capteurs métaboliques

\section{Résumé}

Sensorique moléculaire du métabolisme en cas d'inflammation induite parl'obésité

Les dysfonctionnements métaboliques et les processus chroniques d'inflammation qu'ils entraînent peuvent rendre la transmission cellulaire des signaux plus irrégulière et provoquer ainsi des troubles persistants de la dynamique du métabolisme et de l'homéostasie immune. Ces maladies sont déclenchées par des stimuli de stress chronique tels qu'une alimentation hypercalorique ou un déséquilibre alimentaire et se manifestent principalement sous forme de troubles métaboliques associés à un état d'inflammation systémique (méta-inflammation). Le nombre de maladies concomitantes et consécutives à de tels dysfonctionnements métaboliques et immunologiques augmente rapidement, de sorte que l'on peut presque parler d'une apparition de type pandémique. La méta-inflammation du tissu adipeux se caractérise par un report massif du bilan énergétique dans les systèmes cellulaires impliqués et par la modulation d'importants processus immunologiques. En cas de troubles métaboliques, les adipocytes comme les macrophages sont à la fois d'importants capteurs et effecteurs cellulaires, ce qui peut aussi s'expliquer par le lien génétique et fonctionnel étroit entre les deux types de cellules. Même le système nerveux central (SNC) dépend intrinsèquement du contrôle de l'homéostasie métabolique. Si l'alimentation provoque une augmentation pathologique du tissu adipeux blanc viscéral, survient alors une réaction inflammatoire, légère dans un premier temps, déclenchée par une anomalie de la libération des adipocytokines et une accélération de la circulation des acides gras libres. A défaut d'une correction de ce trouble dans un délai défini, des cellules immunes s'accumulent dans le tissu adipeux. Une inflammation chronique du tissu adipeux génère une insulinorésistance et déclenche d'autres mécanismes pathophysiologiques. Ceux-ci ont des répercussions systémiques et touchent également le tissu périphérique. Des processus de ce type représentent un nouveau phénomène inflammatoire, car ce ne sont ni des bactéries, ni des virus qui déclenchent la réponse cellulaire, mais des troubles métaboliques persistants.

\section{Einleitung}

Jene zellulären Sensorsysteme und Signalpfade, die an der Erkennung und Verwaltung von Nährstoffen beteiligt sind, stellen in ihrer Gesamtheit die molekulare Stoffwechselsensorik dar. Metabolische Signalprozesse interferieren stark mit immunregulatorischen Systemen, die unter anderem bei der Bekämpfung von Pathogenen zum Einsatz kommen. Diese Wechselbeziehung zwischen Stoffwechselsensorik und zellulären Abwehrmechanismen ist aus einer evolutionären Notwendigkeit entstanden, um das Überleben von Zellsystemen und Organismen zu unterstützen $[1,2]$.

Auch die Speicherung von Energie ist eine essenzielle überlebensstrategische Massnahme, sowohl im Sinne der Aufrechterhaltung der basalen metabolischen Grundversorgung als auch für die Keimabwehr. Sie steht somit im Vordergrund der Stoffwechseldynamik. Wird der Organismus von Pathogenen bedroht, stellt die Einleitung und Aufrechterhaltung der Immunantwort einen metabolisch kostspieligen Mechanismus dar, der unter Mangel- oder Fehlernährungsbedingungen nicht effizient ablaufen kann. Fieber oder Sepsis sind stark energieverbrauchende Prozesse; so führt z.B. die Erhöhung der Körpertemperatur um $1^{\circ}$ Celsius zu einer Zunahme des Kalorienverbrauchs um 7-13\% (9,4 × 10 $0^{6}$ Joule). Ist der Organismus nicht in der Lage, diese Energie bereitzustellen, kollabiert das Immunsystem. Unausgewogene Fastenkuren und Fehlernährung führen ebenfalls zu einer verminderten Immunreaktion [1]. In Tierversuchen an Nagern konnte gezeigt werden, dass eine totale Reduktion des Körperfettes zur Abnahme jener Energiereserven führt, die für eine Immunantwort zur Verfügung stehen. Auch bei Insekten wurde eine drastische Senkung der Vitalität und Überlebensrate unter Mangelernährung beobachtet [3]. Die Aufrechterhaltung der Immunantwort erfolgt, aufgrund des hohen Stellenwerts, auf Kosten anderer, energieaufwendigerer Prozesse wie Reproduktion, Thermoregulation oder Zellwachstum [1, 4-6].

Interessanterweise kann aber auch ein Energieüberfluss, wie er bei Adipösen oder bei Patienten, die am metabolischen Syndrom erkrankt sind, vorhanden ist, die Effizienz der Immunabwehr vermindern. Diese Erkrankungsformen sind von exzessiven Entzündungsreaktionen begleitet, die - je nach Ausprägung - das Immunsystem schwächen können oder es gar zum Erliegen bringen. Ein ausgeglichener Energiefluss und die Aufrechterhaltung der metabolischen Homöodynamik sind für eine korrekte Immunantwort notwendig. Wird die Homöodynamik des Organismus dauerhaft gestört, können metabolische Dysfunktionen chronifizieren und das Ausbrechen systemischer Erkrankungen wie Diabetes mellitus, Hyperlipidämie, Arteriosklerose und Bluthochdruck fördern. 


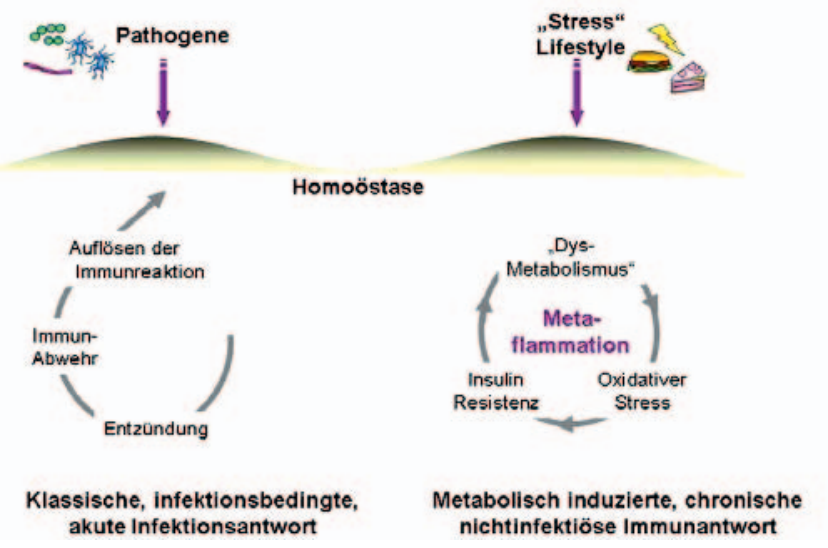

Abb. 1. Übersicht über die Unterschiede zwischen der klassischen pathogeninduzierten Entzündung und der durch metabolische Störungen hervorgerufenen chronisch verlaufenden Metaentzündung (modifiziert nach [14]).

Ein durch Fettleibigkeit induziertes metabolisches Entzündungsphänomen wird als Metaflammation bezeichnet und ist durch besondere Eigenschaften charakterisiert (Abb. 1). Es handelt sich um eine unterschwellige chronische Entzündung, die durch einen Überschuss von Nährstoffen und Energie ausgelöst und von stoffwechselaktiven Zellen koordiniert wird [7].

Ein Beispiel eines Faktors, der traditionell metabolischen Abläufen des Immunsystems zugeordnet wird, ist das von Adipozyten sezernierte Adipozytokin Leptin, das hauptsächlich als Sättigungssensor im Organismus bekannt ist. Während einer frühen Phase der Infektion durch Mikroorganismen korreliert der Status der Infektion mit der Induktion der Leptinsynthese. Die daraus resultierende Senkung der Appetitschwelle wirkt sich negativ auf das Nährstoffangebot, das auch den Pathogenen zur Verfügung steht, aus. Lokal gespeicherte Energie- und Nährstoffressourcen werden zur Aufrechterhaltung der Immunantwort bereitgestellt, ohne das Überleben der Mikroorganismen unnötig zu fördern [1,3]. Mangelnde Bewegung und hyperkalorische Ernährung fördern nicht nur die Ausprägung metabolischer Dysfunktionen mit entzündlichem Charakter, sondern verändern auch das Signalspektrum von Adipozyten und Immunzellen, insbesondere jenes von Makrophagen [5, 8-12]. Adipozyten und Makrophagen sind genetisch verwandt und teilen sich wichtige Aufgaben bei metabolischen und immunologischen Prozessen, wobei es auch funktionelle Überlappungen gibt. Die hier beschriebenen Entzündungsphänomene werden durch metabolische Funktionsstörungen ausgelöst und stellen daher, im Gegensatz zu den klassischen pathogeninduzierten Entzündungen, eine besondere Form der Immunabwehr dar. Die metabolisch induzierte Immunantwort wird durch die Erhöhung des Glukose- spiegels, die Konzentration zirkulierender Lipide, den Anteil an freien Fettsäuren und den Cholesterinspiegel getriggert $[13,14]$.

Weltweit sind derzeit etwa 1,6 Milliarden Menschen übergewichtig; etwa 400 Millionen davon sind fettleibig $[15,16]$. Allen Betroffenen gemeinsam ist eine kalorische Dysregulation von Energieaufnahme, Energiespeicherung und Energienutzung, die zur Initiation oder Prolongation von Entzündungserkrankungen führt. Für alle klinisch relevanten chronischen Stoffwechselkrankheiten, wie Adipositas, Diabetes mellitus Typ 2, Fettleber und Arteriosklerose, konnte eine Verbindung zwischen metabolischer Entgleisung und Entzündung gezeigt werden [5, 9, 10, $16,17]$.

Es ist daher essenziell, auf den derzeitigen Erkenntnisstand über die molekularen Zusammenhänge nutrisensorischer Ereignisse aufmerksam zu machen und neue Ernährungsrichtlinien zu erarbeiten, die einen Beitrag zur Weltvolksgesundheit leisten können.

\section{Zentrales Nervensystem als Schaltstelle des Energie- und Stoffwechselgleichgewichts}

Die Aufrechterhaltung einer energetischen Homöodynamik ist nur durch eine enge Verknüpfung von humoralen Systemen mit dem Zentralen Nervensystem (ZNS) möglich, da nur durch schnelle Kommunikation und Reaktion der Einfluss kurzzeitiger Fluktuationen in der Energieversorgung minimiert werden kann. Insbesondere das vegetative ZNS ist für die Regulierung der Vitalfunktionen und die Aufrechterhaltung der inneren Homöodynamik zuständig. Der Hypothalamus, als primäre Schaltstelle dieses Systems, ist im Speziellen für die Regulation der Nahrungs- und Wasseraufnahme, der zirkadianen Rhythmik und der Thermogenese verantwortlich und koordiniert komplexe neuroendokrine und homöodynamische Funktionen $[18,19]$.

Stress kann dieses System massgeblich beeinflussen. Stress kann definiert werden als ein Zustand, der die Homöodynamik gefährdet und eine Reihe von physiologischen Reaktionen und Verhaltensimpulsen aktiviert. Sowohl intrinsische als auch extrinsische Kräfte (Stressoren) können geordnete Stoffwechselprozesse stören [6,20]. Entzündlich-immunologische Reize wie Infektionen, aktive Autoimmunprozesse, Entzündungsprozesse oder auch Traumata, welche die Achse des sympathischen Nervensystems aktivieren, sind nur einige wenige Beispiele für Stressoren [4, 21].

Um auf Stressereignisse akut und umfassend zu reagieren, bedarf es einer intakten neuroendokrinen, zellulären und molekularen Infrastruktur. Die Koordination der Reaktionen erfolgt vorwiegend durch das sogenannte 
Stresssystem, das sowohl im ZNS als auch in der Peripherie lokalisiert ist. Die zentrale Kontrollstation des Stresssystems ist im Hypothalamus und im Stammhirn angelegt und umfasst die parvozellulären $\mathrm{CRH}$ (corticotropin-releasing hormone)- und AVP(arginin-vasopressin)-Neuronen des hypothalamischen paraventrikulären Kerns (PVN) und das Locus coeruleus(LC)-Norepinephrinesystem (sympathisches Nervensystem). Die HypothalamusHypophysen-Nebennieren-Achse (HPA) und deren assoziierte efferente Systeme stellen die Effektorachse dar, durch die das Gehirn in der Stressphase mit den unterschiedlichen Organen und dem Gewebe kommuniziert [6].

Im Falle einer akuten Aktivierung dieses Systems kommt es zu einer zeitlimitierten Veränderung diverser Körperfunktionen. Die Gesamtheit dieser Veränderungen wird kollektiv mit dem Begriff Stresssyndrom bezeichnet [6]. So wird beispielsweise während einer Stresssituation die Aufmerksamkeit gefördert; das Gehirn fokussiert auf die wahrgenommene Bedrohung. Die Blutauswurfleistung des Herzens nimmt zu und die Atemfrequenz steigt $[5,6]$. Katabole Prozesse werden favorisiert, während gleichzeitig Signale, die zum Appetitverlust und somit zur Nahrungsrestriktion führen, ausgesendet werden und der Blutfluss $\mathrm{zu}$ einer temporär gesteigerten Versorgung von Gehirn, Herz und Muskeln umgeleitet wird [3, 6, 20]. Diese im Normalfall nur vorübergehenden Veränderungen erlauben eine Steigerung des individuellen Überlebens, indem rasch grosse Energiemengen für die Erhöhung der Körpertemperatur und der Intensivierung der Immunantwort zur Verfügung gestellt werden [3, 6, 21].

Chronischer Stress stellt eine anhaltende Bedrohung der Homöodynamik dar, die progressiv zu einer schädlichen Überlastung führt [6]. Ausschlaggebend ist sowohl die persistierende Anwesenheit von Stressoren als auch die dadurch vermittelte prolongierte Aktivierung des Stresssystems. Diese anhaltende Stoffwechselverschiebung führt zu einem generalisierten katabolen Zustand. Eine Vielzahl kürzlich durchgeführter Studien weist darauf hin, dass Erkrankungen wie viszerale Adipositas, Diabetes mellitus Typ 2, Arteriosklerose und metabolisches Syndrom häufig mit chronischem Stress assoziiert sind [4-6]. Die mit chronischem Stress assoziierte milde Hypercortisolämie und die anhaltende Aktivierung des sympathischen Nervensystems begünstigt die Anhäufung von viszeralem Fett und das Entstehen von kardiometabolischen Komplikationen, die das Krankheitsbild von viszeraler Adipositas und Diabetes mellitus Typ 2 prägen. Umgekehrt unterstützt die Fettleibigkeit den systemischen Entzündungsstatus durch eine erhöhte Adipozytokinsekretion, was wiederum zur chronischen Stimulierung des Stresssystems führt [22].

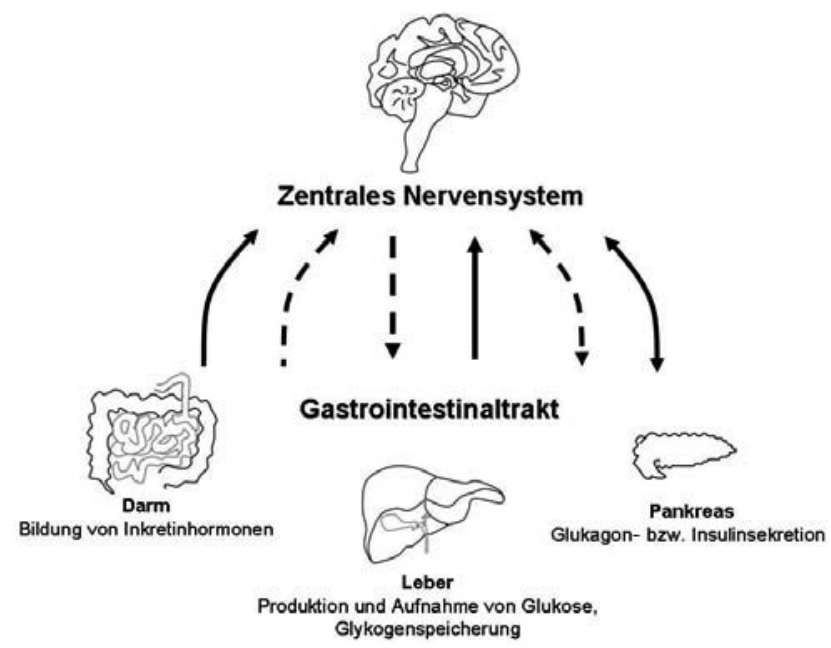

Abb. 2. Zwischen dem ZNS und der Peripherie (Gastrointestinaltrakt, Skelettmuskel) erfolgt ein ständiger, wechselseitiger Austausch von Information. Sowohl Peptidhormone als auch Nährstoffe selbst können dabei direkt (Linie) oder indirekt (unterbrochene Linie) als Signale wirken und zur Regulation der Glukose- und Energiehomöodynamik beitragen.

\section{Beeinflussung der Energiebalance durch \\ Peptidhormone der Peripherie und des ZNS}

Neuronale Schaltkreise müssen die von Nährstoffen und Hormonen ausgesendeten Signale erkennen und darauf koordiniert antworten, um den peripheren Metabolismus zu kontrollieren (Abb. 2). Das ZNS ist in der Lage, komplexe metabolische Prozesse wie die Glukoseaufnahme, die Sekretion von Insulin, die hepatische Glukoneogenese und den Lipidstoffwechsel zu steuern und an veränderte Energiezustände anzupassen, und greift somit in die Regulation der Nahrungsaufnahme, des Energieverbrauchs und der Bewegung ein. Die Signale, welche die neuronalen Schaltkreise beeinflussen können, sind vielfältig und umfassen sowohl periphere als auch zentral vom Hypothalamus sezernierte Neuropeptide und Hormone wie Insulin, GLP-1 (glucagon-like peptide-1), GIP (gastric inhibitory polypeptide), Leptin, CCK (Cholecystokinin), Ghrelin sowie auch Nährstoffsignale selbst, z.B. freie Fettsäuren (free fatty acids, FFA) [19].

Insulin ist ein Peptidhormon, das von den $\beta$-Zellen des Pankreas als Reaktion auf die Zunahme des Blutglukosespiegels freigesetzt wird und diesen durch die Hemmung der hepatischen Glukoneogenese und Förderung der peripheren Glukoseaufnahme reduziert. Im ZNS agiert Insulin als Alarmsignal, das in Folge zu einer Reduktion der Nahrungsaufnahme führt. Insulin kontrolliert das Körpergewicht und den peripheren Glukose- und Fettstoffwechsel; dabei hält es aber nicht nur die Hyperglykämie in Schach, sondern wirkt auch antientzündlich [8, 23]. 
Die Inkretinhormone GLP-1 und GIP werden von den $\mathrm{K}$ - und den L-Zellen im gastrointestinalen Trakt und in geringeren Mengen auch vom ZNS innerhalb von Minuten nach der Nahrungsaufnahme sezerniert. Beide Peptide binden an Rezeptoren, die von Leber- und Skelettmuskelzellen sowie von den $\beta$-Zellen der Langerhans-Inseln im Pankreas exprimiert werden, und stimulieren die Insulinsekretion bei gleichzeitiger Hemmung der Glukagonfreisetzung [24]. CCK wird im Magen-Darm-Trakt gebildet und hat eine wichtige Funktion als Neurotransmitter durch seine Wirkung als Sättigungssignal. CCK kontrolliert die Expression von G-Protein-gekoppelten Rezeptoren und Peptidneurotransmittern in vagal afferenten Neuronen. Wenn die CCK-Plasmakonzentration sinkt, z.B. während einer Phase des Fastens, exprimieren die Neuronen verstärkt CB1(cannabinoid receptor type 1)- und $\mathrm{MCH}$ (melanin-concentrating hormone)-Rezeptoren, die beide mit der Stimulation der Nahrungsaufnahme assoziiert sind [23]. Die Menge an CART (cocaine- and amphetamine-regulated transcript), einem Neuropeptid mit sättigender Wirkung, nimmt hingegen ab. Die CCK-Freisetzung ist abhängig vom Leptin; in Leptin-defizienten Mäusen ist das durch CCK erzeugte Sättigungsgefühl dementsprechend deutlich abgeschwächt [19]. CCK, Ghrelin und Peptid YY (PYY) regulieren wichtige gastrointestinale Funktionen wie Motiliät, Sekretion und Adsorption. PYY hat eine appetitreduzierende Wirkung, während Ghrelin stimulierend wirkt. Die Bildung von Ghrelin erfolgt vermehrt in Zeiten des Fastens und wird nach Einnahme einer Mahlzeit reduziert. Ghrelin beeinflusst die Adipogenese und vermutlich auch den Adipozytenstoffwechsel. Zusätzlich stimuliert es die Produktion von Endocannabinoiden und $\mathrm{MCH}$ [25].

Adiponektin ist eines der am besten untersuchten Adipozytokine. Grosse Mengen an Adiponektin finden sich sowohl im Fettgewebe als auch im Serum. Adiponektin wird ausschliesslich von Fettzellen sekretiert und stellt einen wichtigen Regulator des Energie- und Lipidstoffwechsels dar. Im Unterschied zu anderen Adipozytokinen hat es zudem antientzündliche wie auch antiatherogene Eigenschaften. Ausserdem wirkt Adiponektin Insulin-sensibilisierend, was zumindest teilweise auf die Aktivierung der AMP-aktivierten Proteinkinase (AMPK) und die damit assoziierte Erhöhung von Fettsäureoxidation und Glukoseaufnahme zurückzuführen ist. Bei Insulinresistenz, Diabetes mellitus Typ 2 und Adipositas sind die Adiponektin-Serumlevel reduziert [26].

Insbesondere viele der endogenen peripheren Peptide, die vor, während oder nach der Nahrungsaufnahme ausgeschüttet werden, sind potenziell von pharmakologischem Interesse. Genaue Analysen zur Funktion und zur Wechselwirkung solcher Faktoren stehen daher im Zentrum der medizinischen Forschung.

\section{Nährstoffsensing im Hypothalamus}

Zusätzlich zur Fähigkeit bestimmter Gehirnregionen, im Speziellen des Hypothalamus, eine Vielzahl neuronaler und hormoneller Signale zu integrieren, sind spezialisierte Populationen von Neuronen in der Lage, auf Konzentrationsänderungen zirkulierender Nährstoffe zu reagieren [6]. Der Hypothalamus und insbesondere der basal liegende Nucleus arcuatus sind nahe an der Eminentia mediana lokalisiert - eine Region, in der die Blut-Hirn-Schranke diskontinuierlich verläuft und die somit eine Nahtstelle zwischen Nerven-, Hormon- und Nährstoffsystem darstellt [27].

Glukosesensitive Neuronen im Hypothalamus reagieren mit Änderungen der Frequenz der Aktionspotenziale auf Veränderungen der Blutglukosekonzentration: Glukoseangeregte Neuronen erhöhen die Feuerrate bei zunehmender extrazellulärer Glukosekonzentration, während glukoseinhibierte Nervenzellen die Feuerrate steigern, sobald die Glukosekonzentration abnimmt $[27,28]$. Beide Arten von Neuronen finden sich weit verteilt im Gehirn und in hoher Konzentration in den hypothalämischen Nuclei, die in die Kontrolle der Energiehomöostase involviert sind [6].

Die neuronalen Schaltkreise des Nucleus arcuatus gehören zu den am besten untersuchten Systemen, die eine zentrale Rolle in der Regulation der Energiehomöostase innehaben. Die beiden entgegengesetzt arbeitenden Neuronenpopulationen, die AgRP/NPY(Agouti-related peptide/neuropeptide Y)-exprimierenden und die POMC (pro-opiomelanocortin)/CART-exprimierenden Neuronen, sind prototypische Beispiele für glukosesensitive Neuronen. Die POMC/CART-Neuronen bilden POMC, ein Vorläuferpeptid, das zelltypabhängig zu unterschiedlichen bioaktiven Peptiden prozessiert werden kann. $\mathrm{Zu}$ diesen Peptidhormonen gehören die MSH (melanocytestimulating hormones). $\alpha$ - und $\beta$-MSH regen die Melanocortinrezeptoren 3 und 4 (MC3R/MC4R) an und reduzieren die Nährstoffaufnahme, während sie den Energieaufwand erhöhen. AgRP/NPY-Neuronen bilden NPY, das die Nahrungsaufnahme stimuliert und den Energieverbrauch reduziert. AgRP wirkt als inverser Agonist auf MC3R und MC4R und verhindert den anorektischen Effekt von $\alpha$ MSH. Nimmt die extrazelluläre Glukosekonzentration zu, werden AgRP/NPY-Neuronen inhibiert und POMC-Neuronen angeregt [27].

Auch FFA gelangen in das ZNS. Die Aufnahmemenge ist dabei abhängig von der FFA-Konzentration im Blut, da der Grossteil der FFA durch Diffusion über die Blut-HirnSchranke gelangt. Hohe Konzentrationen von zirkulierenden FFA sind unter anderem für das Krankheitsbild der Adipositas kennzeichnend [18, 27]. Ein kleiner Teil der Fettsäureaufnahme im Gehirn erfolgt wahrscheinlich auch 
durch direkte Lipoproteinrezeptor-vermittelte Internalisierung von Lipoproteinpartikeln. In der Zelle werden Fettsäuren rasch von dem Enzym Acyl-CoA-Synthetase (ACS) zu Acyl-Coenzym A (Acyl-CoA) verestert und in den zellulären Acyl-CoA-Pool integriert. Die weitere Prozessierung, z.B. durch Oxidation oder durch Einbau in Phospholipide, ist abhängig von der Art der Fettsäure. FFA können im ZNS aber auch als zelluläre Botenstoffe agieren und wie die Glukose als metabolische Sensoren dienen. Es konnte gezeigt werden, dass eine Hemmung der hypothalamischen Fettsäuresynthase zum Absenken von Nahrungsaufnahme, hepatischer Glukoneogenese und Insulinkonzentration führt $[19,27,29]$. Lipidsensitive Neuronen im Hypothalamus können ihre Freisetzungsrate in Abhängigkeit von der Konzentration der FFA modifizieren. Diese Effekte sind auch glukoseabhängig; die genauen molekularen Mechanismen sind jedoch noch nicht bekannt. Es gibt allerdings Hinweise, dass Fettsäuren die Leitfähigkeit von unterschiedlichen Ionenkanälen beeinflussen [27].

\section{Stress, metabolisches Ungleichgewicht und Entzündung}

Das zunehmende Verständnis der Relation von Stress, metabolischem Gleichgewicht und Entzündung gibt neue Einblicke in die Pathophysiologie metabolischer Komplikationen. Der moderne Mensch ist einer chronischen Stressbelasung ausgesetzt; dies betrifft sowohl den sozialen Bereich als auch, aufgrund der ständigen Überernährung, den Gesamtstoffwechsel.

Während der katabole Status und die Suppression des Immunsystems bei temporär begrenztem, akutem Stress einen Vorteil für den Gesamtorganismus bringt, ist eine chronische Hyperaktivierung der HPA-Achse durch das Auftreten diverser somatischer Störungen charakterisiert, die nicht nur die Lebensqualität beeinflussen, sondern sich auch auf die Lebenserwartung negativ auswirken. Klinische Manifestationen von chronischem Stress zeigen ein breites Spektrum an Folgeerkrankungen wie Osteroporose, Merkmale des metabolischen Syndroms (z.B. viszerale Fettanlagerungen, gestörte Glukosetoleranz, Dyslipidämie, Bluthochdruck), arteriosklerotische Veränderungen, Immunsuppression und somit häufigeres Auftreten von Infektion bis hin zu neoplastischen Veränderungen. Die Zunahme an Stress korreliert auch mit erhöhten Inzidenzraten von Adipositas und metabolischem Syndrom [1, 3, $4,9]$.

Bestimmte Arten von chronischem Stress resultieren in einem Pseudo-Cushing-Status, der wahrscheinlich einem durch Stress induzierten, milden Hypercortisolismus und einer erhöhten Glucocorticoidsensitivität peripherer Gewebe zugeschrieben werden kann. In einigen Fällen von viszeraler Adipositas findet man aber auch eine Unter- drückung der Sekretion von Cortisol, Wachstumshormonen und Sexualsteroidhormonen. Dieser durch Hyperaktivierung des sympathischen Nervensystems begleitete Status kennzeichnet möglicherweise ein Burnout der Reserven der HPA-Achse [6].

Fettleibigkeit ist generell von leichten, aber persistierenden systemischen Entzündungsprozessen begleitet. Dieser entzündliche Stress wird unter anderem durch die Sekretion von proinflammatorischen Zytokinen unterhalten, die vom adipösen Gewebe ausgeht. Insbesondere Tumornekrosefaktor alpha (TNF- $\alpha$ ), Interleukin 6 (IL-6) und Monozyten-chemotaktisches Protein 1 (MCP1, auch CCL2) werden in stark erhöhten Mengen gebildet. TNF trägt neben seinen immunmodulatorischen Eigenschaften zur Entwicklung der Insulinresistenz bei, da er die intrazelluläre Signalweiterleitung der Insulinrezeptoren blockiert. IL-6 hat atherogene Eigenschaften und wirkt auf alle wichtigen endokrinen Achsen. Es vermittelt die zentrale Stimulation der Cortisolsekretion in Kombination mit der Unterdrückung der Thyreotropin- und Testosteronsekretion und beeinflusst den Glukose- und Lipidmetabolismus $[6,26]$. Auch andere Adipozytokine wie Resistin, Visfatin, Leptin und die verminderte Sekretion von Adiponektin tragen zum Entzündungsphänomen bei.

Stress und die daraus folgende Stimulierung der lokalen und systemischen Entzündung führt auch zu einer $\mathrm{Zu}-$ nahme der Schmerzsensibilisierung und anderen Defekten [30]. Angelockt durch chemotaktische Proteine wie MCP1 werden Immunzellen in das Fettgewebe rekrutiert. Die Akkumulation von Makrophagen und T-Zellen im Fettgewebe löst eine zusätzliche Produktion von Zytokinen aus. Proentzündliche Mediatoren, die sowohl von Fettgewebs- als auch von Immunzellen abgesondert werden, provozieren die hepatische Produktion von C-reaktivem Protein (CRP). Die Konzentration von CRP im Blut reflektiert den generellen Entzündungszustand. Mehrere Studien konnten zeigen, dass die Zunahme an viszeralem Fettgewebe von einer Erhöhung des Levels an CRP begleitet ist [31].

\section{Entwicklung und Funktion des Fettgewebes}

Bei Normalgewichtigen (ca. $75 \mathrm{~kg}$ bei 1,70 Meter Körpergrösse) macht das Fettgewebe, das zu 95\% aus Triacylglycerinen besteht, etwa $12 \%$ des Körpergewichts aus (ca. $8 \mathrm{~kg}=308000 \mathrm{~kJ}$ ) [32]. Die Speicherung der Energie in Form von Triacylglycerinen bietet gegenüber anderen Energielieferanten, wie Proteinen oder Glykogen, den Vorteil, den Organismus langfristig (über 24 h hinaus) ausreichend mit Energie in Form von Fettsäuren versorgen zu können. Die Energiereserven eines Normalgewichtigen reichen bei einem durchschnittlichen gerechneten Verbrauch von $8400 \mathrm{~kJ}$ aus, um den Energiebedarf des mensch- 


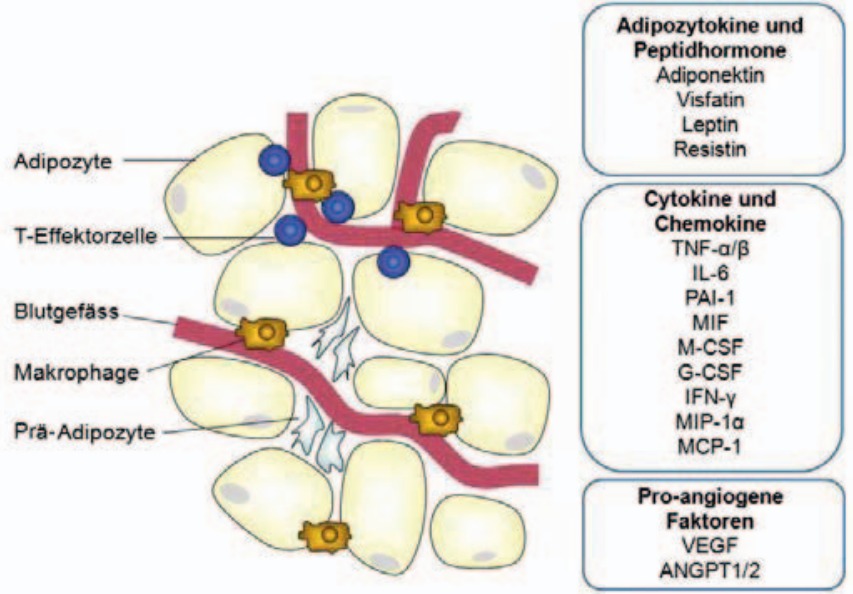

Abb. 3. Das adipöse Fettgewebe zeigt eine Ansammlung von reifen Adipozyten, Prä-Adipozyten, vaskulären Zellen und Immunzellen. Um das Volumen zu vergrössern, werden Faktoren sezerniert, welche die Differenzierung und Proliferation der Adipozyten fördern und die Blutgefässneubildung stimulieren. Adipozytokine sowie der erhöhte Spiegel an freien Fettsäuren führen zur verstärkten Rekrutierung von Makrophagen und anderen Immuneffektorzellen, die wiederum inflammatorische Mediatoren produzieren. Die vom adipösen Fettgewebe gebildeten Signale unterhalten nicht nur lokale, sondern auch systemische Entzündungsprozesse und greifen in den Metabolismus anderer Gewebe ein (modifziert nach [9]).

Abkürzungen: TNF- $\alpha / \beta=$ Tumornekrosefaktor alpha/beta;

IL-6 = Interleukin 6; PAI-1 = Plasminogen-Aktivator-Inhibitor 1;

MIF = Makrophagenmigration-inhibierender Faktor;

M-CSF-1 = Makrophagenkolonien-stimulierender Faktor 1;

G-CSF = Granulozytenkolonien-stimulierender Faktor;

IFN- $\gamma=$ Interferon- $\gamma$; MIP-1 $\alpha=$ Makrophagen-inflammatorisches Protein 1 $\alpha$, auch CCL3; MCP-1 = Monozyten-chemotaktisches Protein, auch CCL2; VEGF = vaskulärer endothelialer Wachstumsfaktor; ANGPT1/2 = Angiopoietin 1/2.

lichen Körpers für 37 Tage zu decken [3, 20, 32, 33]. Das Fettgewebe des erwachsenen Menschen ist das plastischste Gewebe im Körper, da es sich je nach Situation ausdehnen oder schrumpfen kann $[12,16]$.

Grundsätzlich unterscheidet man zwischen dem weissen bzw. gelben, univakuolären, metabolisch inaktiven Fettgewebe und dem braunen, plurivakuolären, metabolisch aktiven Fettgewebe, das von Neugeborenen zur Wärmeproduktion herangezogen wird $[8,16,23,33]$. Beim Erwachsenen ist das braune Fettgewebe nur noch an bestimmten Körperstellen wie Nacken, Mittelfell (Mediastinum) und supraklavikulären Bereichen zu finden [16]. Das weisse Fettgewebe hat neben seiner Schutz- und Stützfunktion die Aufgabe, Energie zu speichern und zu mobilisieren. Im Säuglings- und Kindesalter erfolgt eine maximale Anhäufung von Fettgewebe stets vor einer Wachstumssteigerung [34]. Bis zum Erwachsenenalter verteilt sich das Fettgewebe nach dem androiden abdominalen oder auch viszeralen Muster bzw. nach dem weib- lichen gynoiden Muster, bei dem sich das Fett hauptsächlich an Oberschenkeln und Gesäss anlagert [32, 33].

Köperfettzunahme und die Vergrösserung von abdominalen bzw. viszeralen Fettdepots sind mit einer höheren Prävalenz bzw. Inzidenz für Adipositas und der damit vergesellschafteten kardiovaskulären Erkrankungen assoziiert. Eine viszerale Fettablagerung erfolgt aber erst, nachdem die subkutane Fettablagerungskapazität erschöpft ist [4, 17, 35]. Adipositas kennzeichnet einen Zustand exzessiver Fettspeicherung, der mit gravierenden metabolischen Dysfunktionen assoziiert ist [11]. Laut der Weltgesundheitsorganisation (WHO) sind gegenwärtig 1,6 Milliarden Erwachsene übergewichtig (Body Mass Index/BMI $>25 \mathrm{~kg} / \mathrm{m}^{2}$ ) und mindestens 400 Millionen klinisch adipös (BMI $\left.>30 \mathrm{~kg} / \mathrm{m}^{2}\right)[5,36]$. Bei stark adipösen Personen stellt das weisse Fettgewebe den grössten Anteil an Gewebe dar und ist am ganzen Körper verteilt [16].

Das adipöse Fettgewebe ist eine integrative, metabolisch aktive Ansammlung von reifen Adipozyten, Prä-Adipozyten, vaskulären-, neuralen- und Immunzellen und kann auch als das grösste endokrine Organ angesehen werden (Abb. 3). Hier werden multiple Signale generiert, die regulatorisch in den Metabolismus anderer Gewebe eingreifen $[12,23]$. Adipozyten passen sich in Zeiten exzessiver Kalorienzufuhr durch Umformung an, um für die erhöhte Triacylglyceridspeicherung in Form von Lipidtröpfchen Platz zu schaffen [23]. Obwohl dieser Speichermechanismus evolutionsbedingt ist, kann die Adipozytenfunktion durch eine chronisch positive Energiebilanz (Energiezufuhr $>$ Energiebedarf) gestört werden [12]. Nicht nur die Zunahme der Grösse der Fettzellen selbst (Hypertrophie), sondern auch die Zunahme der Anzahl (Hyperplasie) der Adipozyten trägt dazu bei, dass der Anteil an Fettgewebe bei Überernährung zunimmt $[35,37]$.

Sobald das kritische Volumen der im Fettgewebe ansässigen Adipozyten erreicht ist, werden Signale ausgeschüttet, um neue Prä-Adipozyten zu rekrutieren und um Proliferation und Differenzierung zu fördern. Dadurch nimmt das Ausmass der Adipozytenhypertrophie und Hyperplasie noch weiter zu [35, 37]. Hypertrophe Adipozyten produzieren unter In-vitro-Bedingungen Faktoren, die in parakriner Weise diesen Kreislauf unterhalten, wie z.B. TNF und IGF-1(insulin-like growth factor 1). Im Gegensatz zu anderen Fettgewebsarten besitzt das weisse viszerale Fettgewebe zudem eine sehr hohe Anzahl an Glucocorticoid- und $\beta$-Adreno-Rezeptoren $[4,5]$. Auch zirkulierende Glucocorticoide, Wachstumshormone sowie Insulin scheinen die Prä-Adipozytendifferenzierung zu beeinflussen [12].

Der Status des Prä-Adipozyten wird durch die Expression des löslichen Proteins Pref-1 (preadipocyte factor 1) aufrechterhalten. Im Laufe des Differenzierungsprozesses zum reifen Adipozyten nimmt die Konzentration an Pref-1 ab, während spezifische Transkriptionsfaktoren 
sequenziell aktiviert werden [23]. $\mathrm{Zu}$ den Adipogeneseregulierenden Faktoren gehören C/EBP (CCAAT/enhancer-binding protein) $-\alpha,-\beta$ und $-\gamma$, PPAR $\gamma$ (peroxisome proliferator-activated receptor gamma) und SREBP-1/ ADD-1 (sterol regulatory element-binding protein 1/adipocyte determination and differentiation-dependent factor 1) $[33,34,38]$. Erkennungsmerkmale eines ausdifferenzierten Adipozyten sind die Expression von Genen, die an der Lipogenese beteiligt sind, wie FAS (fatty acid synthase), und das Vorhandensein des insulinabhängigen Glukosetransporters-4 (GLUT-4), der nach Stimulation die Fusion zwischen den Speichervehikeln und der Plasmamembran fördert und die Aufnahme von Glukose ermöglicht [23]. Voll ausdifferenziertes Fettzellgewebe hat die notwendige Insulinsensitivität entwickelt und besitzt die Fähigkeit zur Sekretion und Produktion von Faktoren wie Leptin, PAI-1 (plasminogen activator inhibitor-1) oder Adiponektin [34] Morphologisch ist der Differenzierungsprozess vom PräAdipozyten zum Adipozyten von der Bildung eines univakuolären Lipidtröpfchens (Vakuole) und eines vaskulären Systems begleitet. Dieses Vakuolensystem ist für die Regulation der Aufnahmefähigkeit immenser Mengen an Lipiden und Cholesterin wichtig, die unter anderem durch das Fettsäure-Carrierprotein FABP4 (fatty acid binding protein 4) und die Lipoproteinlipase (LPL) gewährleistet wird $[23,34]$.

Gesundes Fettgewebe ist auch von einer funktionierenden Neovaskularisation abhängig. Für dessen Bildung wird eine Reihe von Faktoren sekretiert, zu denen auch ANGPT1/2 (angiopoietin 1/2) und VEGF (vascular endothelial growth factor) gehören. VEGF spielt nicht nur bei der Vaskularisierung des Fettgewebes, sondern auch bei der Adipogenese eine wichtige Rolle [16, 23]. Treten Fehler in der Entwicklung einer adäquaten Fettmasse auf, spricht man von Lipodystrophie, während man bei einem absoluten Fehlen der Fettmasse von Lipoatrophie spricht [38].

Sowohl unter physiologischen als auch unter pathologischen Bedingungen wird vom Fettgewebe eine Reihe von Faktoren (unter anderem Adipozytokine, Peptidhormone) sezerniert, die für regulatorische Funktionen anderer Organe und Zellen wichtig sind [17]. Das Fettgewebe in seiner Funktion als endokrines Organ trägt im gesunden Organismus zur Aufrechterhaltung der metabolischen und immunologischen Homöodynamik bei. Es beeinflusst den Substratstoffwechsel, reguliert das Sättigungsgefühl usw. $[11,13,15,16]$. Die endokrinologischen Funktionen des Fettgewebes haben einen direkten Einfluss auf die Pathophysiologie der Adipositas, des metabolischen Syndroms und der daraus folgenden kardiovaskulären Komplikationen. Exzessive Konzentrationen an zirkulierendem Cholesterin, Triacylglycerin und FFA verursachen zelluläre Dysfunktionen, die grossteils durch eine Fehlsteuerung des Lipidstoffwechsels und der damit einhergehenden Bildung von lipotoxischen Substanzen entstehen [16,
17, 32]. Die Veränderungen im Differenzierungs- und Sekretionsverhalten der Adipozyten provozieren einen chronisch entzündlichen Status, der auch andere Gewebe und Organe angreift. Ektope Lipidablagerungen in nicht adipozytärem Gewebe wie dem Skelett- oder Herzmuskel führen zu peripherer Insulinresistenz und endothelialen Fehlfunktionen [35].

Das Fettgewebe selbst kann im adipösen hypertrophen Zustand nicht mehr genügend mit Sauerstoff versorgt werden und ist somit hypoxischen Bedingungen ausgesetzt $[16,39]$. Hypoxie trägt dazu bei, dass proinflammatorisch wirksame Adipozytokine produziert werden, und verstärkt somit die Entzündung. Dieser Response auf niedrige Sauerstoffniveaus erfolgt durch die Aktivierung spezifischer Transkriptionsfaktoren, zu denen auch HIF-1 (hypoxia-inducible factor 1) gehört. Das Schlüsselelement dieses heterodimeren Transkriptionsfaktors ist die HIF$1 \alpha$-Untereinheit, die als molekularer Sauerstoffsensor bezeichnet werden kann. Während HIF-1 $\alpha$ unter normalen Sauerstoffbedingungen sofort nach der Synthese proteasomal degradiert wird, wird der Faktor bei Hypoxie stabilisiert und bildet mit der zweiten Untereinheit HIF-1 $\beta$ den aktiven Transkriptionsfaktor. HIF-1 ist an der Transaktivierung von über 70 verschiedenen Genen beteiligt, die in Prozesse der Angiogenese, des Glukosemetabolismus, der Apoptose, des Zellstresses, des Remodellings der extrazellulären Matrix und der Entzündung involviert sind [39]. Hypoxie induziert extensive Veränderungen in den Adipozyten, vor allem, was die Expression und Freisetzung von entzündungsrelevanten und proangiogenen Faktoren betrifft. Erhöhte mRNA-Spiegel von GLUT-1, IL-6, Leptin, PAI-1, MIF (macrophage migration inhibitory factor) und VEGF wurden beobachtet, während die Adiponektinsekretion in hypoxischen Adipozyten abnimmt $[16,23,38]$.

\section{Fettgewebsentzündung}

Das Fettgewebe ist, wie andere metabolisch aktive Gewebe und Organe, in der Lage, immunologische Zellantworten mit $\mathrm{zu}$ beeinflussen und zu kontrollieren [1, 12]. Diese Verbindung zwischen Metabolismus und Entzündung ist evolutionär bedingt und wird auch durch die räumliche Nähe von Immunzellen und metabolisch aktivem Gewebe verdeutlicht. Ins Fettgewebe eingebettete Lymphknoten stellen eine direkte Schnittstelle zum Immunsystem dar. Möglicherweise haben diese Lymphkoten auch einen «Wettbewerbsvorteil» in Zeiten von limitierter Energieversorgung [1].

Wird das Lipidspeicher- und Prozessierungspotenzial der Adipozyten überfordert, kommen Stresserscheinungen zum Tragen, wie hypertrophe Proliferation und der damit verbundene mechanische Stress, Veränderungen der $\mathrm{Zu}$ sammensetzungen der Lipide und anderen Nährstoffen, 
Hypoxie, Störungen der Mitochondrienfunktion und ROS(Reactive Oxygen Species)-Bildung, Apoptoseinduktion, erhöhte Freisetzung von Fettsäuren, ER-Stress und Veränderungen der Adipozytokin-Signalweiterleitung. Die Interaktion der Adipozyten bzw. der sezernierten Faktoren mit den infiltrierenden Immuneffektorzellen ist entscheidend für das Auslösen einer adipösen Fettgewebsentzündung (Abb. 3) [11, 15, 38].

Die Adipozytokine Adiponektin, Leptin, Resistin und Visfatin nehmen eine Schlüsselrolle in der Verbindung von Fettleibigkeit, Insulinresistenz und den damit assoziierten entzündlichen Erkrankungen ein. Adipozytenhypertrophie und der damit verbundene Stress aktivieren metabolische Stoffwechselwege, die erst zur Insulinresistenz im Adipozyten selbst führen. Dieser Status ist charakterisiert durch eine niedrige Liposynthese und eine erhöhte Lipolysekapazität, was mit einem erhöhten Spiegel an zirkulierenden FFAs korreliert [37, 38]. Im Laufe der Fettgewebsentzündung breitet sich die Insulinresistenz auf das gesamte Fettgewebe und auch auf periphere Organe aus. Insulin, das von Pankreas- $\beta$-Zellen sezerniert wird, hat die Aufgabe, in insulinsensitiven Geweben (Leber, Skelettmuskel, Fettgewebe und auch Makrophagen) die Aufnahme, Oxidation und Speicherung von Energielieferanten $\mathrm{zu}$ regulieren. Insulinresistente Gewebe zeigen eine verminderte Reaktion auf die insulinvermittelte Verwertung von Glukose und Fettsäuren. Insulinresistenz tritt oft gleichzeitig mit Hyperinsulinämie und Hyperglykämie auf und ist mit einem erhöhten Risiko von Diabetes mellitus Typ 2 assoziiert $[9,37]$.

Jedoch sind auch andere Faktoren wie TNF, IL-6, MCP1, PAI-1 und bestimmte Komplementfaktoren prägend für das Entzündungsphänomen im Fettgewebe $[9,11,12]$. Alle diese Faktoren tragen dazu bei, dass die im Fettgewebe entstandenen Entzündungsereignisse dauerhaft bestehen bleiben. Die Fettgewebsentzündung bleibt nicht nur lokal, sondern breitet sich systemisch aus und betrifft auch andere Gewebe und Organe. Dieses durch eine metabolische Dysfunktion hervorgerufene Entzündungsphänomen ist mit der Entwicklung einer Reihe von Begleiterkrankungen, wie vaskulär-endothelialen Dysfunktionen, abnormen Lipidprofilen, Bluthochdruck und Gefässentzündungen, assoziiert [15, 38].

Die Induktion der Expression von entzündungsrelevanten Genen im Fettgewebe geht mit einer vermehrten Makrophagen- und T-Lymphozyteninfiltration einher (Abb. 3) [12, 13, 38]. Der Prozentsatz der Makrophagen im Fettgewebe korreliert dabei direkt mit dem Ausmass der Adipositas und der Grösse der Adipozyten [12, 36]. Die vermehrte Sekretion von Leptin und MCP-1 im adipösen Fettgewebe trägt ebenfalls zur Rekrutierung von Effektorzellen bei. Monozyten exprimieren Leptin- sowie MCP-1Rezeptoren (CCR-2, chemokine (C-C motif) receptor 2) und werden durch deren Aktivierung zur Sekretion von
Entzündungsmediatoren stimuliert $[5,10,13]$. Auch andere Signale wie M-CSF (macrophage colony stimulatig factor, auch CSF-1), G-CSF (granulocyte colony stimulating factor) und MIF werden vom adipösen Fettgewebe sezerniert und tragen zur Immunzellrekrutierung und Differenzierung bei $[23,38]$.

Die vermehrte Anwesenheit von Immuneffektorzellen stört die Adipozytenhomöodynamik, da die Sekretion von proinflammatorischen Zytokinen die Immunreaktion zusätzlich triggert [12]. Erhöhte Spiegel an IFN- $\gamma$ (Interferon- $\gamma$ ) im Fettgewebe deuten auf eine erhöhte Aktivität von chemotaktisch aktiven Entzündungsmediatoren hin. Im Tierversuch konnte gezeigt werden, dass IFN- $\boldsymbol{\gamma}$-defiziente Mäuse im adipösen Zustand eine reduzierte Produktion entzündlicher Zytokine sowie Makrophagenrekrutierungsrate und eine verbesserte Glukosetoleranz aufwiesen [25]. Des Weiteren setzen infiltrierende Makrophagen Entzündungsmarker wie MCP1, IL-6, TNF- $\alpha$, TGF $\beta$, MIP-1 $\alpha$ (macrophage inflammatory protein $1 \alpha$, auch CCL3) und iNOS (inducible nitric oxide synthase) frei $[13,17,37]$. Dadurch werden einerseits weitere Immunzellen rekrutiert, während andererseits die in den Adipozyten stattfindende Lipolyse stimuliert wird, was die vermehrte Freisetzung von nichtveresterten freien Fettsäuren (NEFA) zur Folge hat $[13,38]$.

Makrophagen und Adipozyten sind nicht nur beide in metabolische und immunologische Mechanismen eingebunden, sondern beide Zelltypen weisen ein verwandtes Expressionsprofil, insbesondere im Hinblick auf metabolische Gene, auf und sind sich im differenzierten Zustand auch funktionell sehr ähnlich. Die funktionelle Überlappung betrifft nicht nur die Sekretion von Zytokinen und Adipozytokinen, sondern auch die Fähigkeit zur Reaktion auf pathogenassoziierte Komponenten wie Lipopolysaccharide (LPS), das Phagozytose-Verhalten und die Fähigkeit zur Expression von membrangebundenen NADPHOxidasen [1]. Bei den im adipösen Gewebe ansässigen Makrophagen handelt es sich um M1- und M2-Makrophagen. Die Einteilung in M1 und M2 nach Mills et al. [40] beschreibt Populationen von Makrophagen, die unterschiedliche metabolische Programme exprimieren. Sie beeinflussen die Lymphozytenproliferation sowie die Zytokinproduktion und lenken somit den Immunresponse. M1 oder «klassisch aktivierte» Makrophagen werden durch Stimulation mit proinflammatorischen Mediatoren, wie bakteriellen LPS und IFN- $\gamma$, aktiviert. Sie produzieren proinflammatorische Zytokine (z.B. TNF- $\alpha$, IL-6, IL-12) und generieren ROS. M2 oder «alternativ aktivierte» Makrophagen bilden proinflammatorische Zytokine in viel geringerem Ausmass; sie exprimieren jedoch grosse Mengen an antiinflammatorischem IL-10- und IL-1«Decoy»-Rezeptor. Zudem weisen sie eine erhöhte Arginaseproduktion auf. M2-Makrophagen können eine inflammatorische Reaktion blockieren und beeinflussen die 
Gewebsregeneration positiv [40, 41]. Eine differenzielle Aktivierung der Makrophagen in Abhängigkeit vom Ausmass der Adipositas scheint wahrscheinlich. Lumeng et al. beschrieben im Tiermodell eine erhöhte Anzahl von proinflammatorischen M1-Zellen sowie den Verlust der protektiven Kapazität von M2-Makrophagen in ernährungsbedingt induzierter Fettleibigkeit [13, 41].

Die Kommunikation von Makrophagen und Adipozyten im Fettgewebe und peripheren Gewebe durch parakrine Mediatoren bewirkt, dass sich anfänglich lokale Entzündungsphänomene zu systemischen Defekten umwandeln. Dies zeigt sich beispielsweise bei der Insulinresistenz. Aufgrund dieser Erkenntnisse gibt es Ansätze, durch die Supprimierung der Fettgewebsentzündung die Behandlung der Adipositas und der damit verbundenen metabolischen Störungen zu unterstützen [38].

\section{Schlussfolgerung: Rolle der Entzündung bei metabolischen Erkrankungen}

Das Fettgewebe stellt mehr als ein Speicherorgan für überschüssige Energiereserven dar. Es beeinflusst durch seine metabolischen und immunologischen Funktionen den Stoffwechsel des Gesamtorganismus. Das Zusammenspiel parakriner Mediatoren mit dem ZNS ermöglicht es, bei kurzzeitigen Anforderungen eines erhöhten Energiebedarfs adäquat zu reagieren. Bei ständig erhöhter Energiezufuhr werden die Speicherkapazitäten der Adipozyten überfordert und die zelluläre Homöodynamik gestört. Diese Dysbalance verursacht molekulare Veränderungen, die sich nicht nur zellulär, sondern auch systemisch auswirken. Die Dysregulation des Metabolismus in der Adipositas betrifft unterschiedliche Organsysteme und erhöht die Prävalenz von Insulinresistenz und Dyslipidämie, was in Folge die Entwicklung von Diabetes und kardiovaskulären Erkrankungen fördert. Auch Krankheiten wie rheumatoide Arthritis, chronische Darmentzündung und zystische Fibrose, die durch einen permanenten Entzündungszustand charakterisiert sind, sind mit einer frühzeitigen und/oder schweren metabolischen Dysfunktion assoziiert $[12,38]$.

Das Fettgewebe kontrolliert die Menge an zirkulierenden FFA und beeinflusst die systemische Homöodynamik durch die Sekretion von Adipozytokinen. Der veränderte Lipidstoffwechsel ist zusammen mit anormalen Entzündungsantworten eine zentrale Ursache für die Entstehung der Arteriosklerose [9, 15]. Im adipösen Fettgewebe werden im Zuge der metabolischen Entgleisung immer mehr Immunzellen, wie T-Zellen und Makrophagen, rekrutiert und ein chronisch entzündliches Milieu geschaffen.

In adipösen Individuen ist die Konzentration proentzündlicher Faktoren erhöht, was zu Gewebsentzündungen, systemischer Entzündung und erhöhtem oxidativen Stress führt [12]. Die Fettgewebsentzündung wird als ursächlich für die Unterhaltung eines chronischen inflammatorischen Status im Gesamtorganismus angenommen. Die Entzündung wird dabei nicht durch Pathogene, sondern durch eine metabolische Dysbalance induziert (Metaflammation), die aufgrund des Lebensstils oder durch Umwelteinflüsse hervorgerufen werden kann, z.B. bei Über- und Unterernährung, Bewegungsmangel, Schlafmangel, Rauchen, Depression oder Schadstoffbelastung usw. [14]. Im Gegensatz zur klassischen Entzündung ist dieses metabolische Entzündungsphänomen mit einer reduzierten Stoffwechselrate assoziiert und stellt aufgrund der dauerhaften Stoffwechselumstellungen eine potenzielle Gesundheitsgefahr dar.

Erste Folgen sind die lokale Insulinresistenz sowie eine unkontrollierte Freisetzung von Fettsäuren, inflammtorischen Zytokinen und Adipozytokinen, was ultimativ auch zu systemischen Effekten führt [7]. Aktuelle Daten weisen darauf hin, dass metabolische Krankheiten auch Veränderungen der intestinalen Flora zur Folge haben können. Beispielsweise gelangt LPS vor allem im Zuge der Lipidabsorption bei der Verdauung in den Organismus. Eine chronisch fettreiche Ernährung oder verschlechterte Barrierefunktionen im Darm führen zu vermehrter Endotoxinämie, was einen zusätzlichen Stimulus für das Entzündungsgeschehen darstellt [7].

Die wissenschaftliche Aufarbeitung der molekularen Stoffwechselsensorik (sogenanntes Nutrisensing) gewinnt zunehmend an Bedeutung. Nutrisensing-Systeme sind zelluläre Erfassungssysteme für die An- oder Abwesenheit von Nährstoffen und übernehmen das zelluläre Nährstoffmanagement. Es handelt sich dabei um evolutionär konservierte Stoffwechselwege und biochemische Mechanismen, die eine zentrale Funktion bei der Aufrechterhaltung der metabolischen Homöodynamik haben und integrativ mit den Sensormechanismen für Pathogene sowie dem Immunsystem wirken [1].

Es besteht eine enge Korrelation zwischen der metabolischen Entgleisung infolge von Mangel-, Fehl- und Überernährung und der Ausprägung von chronischen Entzündungsreaktionen und damit assoziierten Folgeerkrankungen $[1,2,23]$. Erst das Verständnis der komplexen Zusammenhänge der sensorischen Leistungen der Zellen selbst, deren Interaktion und Kommunikation innerhalb des Gewebes, mit dem ZNS und anderen peripheren Organen sowie die Einsicht in die integrative Beziehung von Metabolismus und Immunsystem haben zu einer Neubewertung der Auslösefaktoren metabolisch bedingter Stoffwechselstörungen geführt.

\section{Disclosure Statement}

Die Autoren erklären, dass kein finanzieller oder sonstiger Interessenkonflikt im Zusammenhang mit dem Inhalt des Beitrags besteht. 


\section{Literatur}

1 Hotamisligil GS, Erbay E: Nutrient sensing and inflammation in metabolic diseases. Nat Rev Immunol 2008;8:923-934

2 Yang Z, Kahn BB, Shi H, Xue BZ: Macrophage $\alpha 1$-AMP-activated protein kinase ( $\alpha 1 \mathrm{AMPK})$ antagonizes fatty acid-induced inflammation through SIRT1. J Biol Chem 2010;285:1905119059.

3 Fernández-Riejos P, Najib S, Santos-Alvarez J, Martín-Romero C, Pérez-Pérez A, GonzálesYanes C, Sánchez-Margalet V: Role of leptin in the activation of immune cells. Mediators Inflamm 2010;2010:568343.

4 Shively CA, Register TC, Clarkson TB: Social stress, visceral obesity, and coronary artery atherosclerosis: product of a primate adaptation. Am J Primatol 2009;71:742-751.

5 Brydon L: Adiposity, leptin and stress reactivity in humans. Biol Psychol 2011;86:114-120.

6 Kyrou I, Tsigos C: Stress mechanisms and metabolic complications. Horm Metab Res 2007;39:430-438.

7 Gregor MF, Hotamisligil GS: Inflammatory mechanisms in obesity. Annu Rev Immunol 2011;29:415-445.

8 Laugerette F, Vors C, Peretti N, Michalski MC: Complex links between dietary lipids, endogenous endotoxins and metabolic inflammation. Biochimie 2011;93:39-45.

$\checkmark 9$ Tilg H, Moschen AR: Adipocytokines: mediators linking adipose tissue, inflammation and immunity. Nat Rev Immunol 2006;6:772-783.

10 Dandona P, Ghanim H, Chaudhuri A, Dhindsa S, Kim SS: Macronutrient intake induces oxidative and inflammatory stress: potential relevance to atherosclerosis and insulin resistance. Exp Mol Med 2010;42:245-253.

11 Stofkova A: Resistin and visfatin: regulators of insulin sensitivity, inflammation and immunity. Endocr Regul 2010;44:25-36.

12 Iyer A, Fairlie DP, Prins JB, Hammock BD Brown L: Inflammatory lipid mediators in adipocyte function and obesity. Nat Rev Endocrinol 2010;6:71-82

13 Gutierrez DA, Puglisi MJ, Hasty AH: Impact of increased adipose tissue mass on inflammation, insulin resistance, and dyslipidemia. Curr Diab Rep 2009;9:26-32.

14 Egger G, Dixon J: Inflammatory effects of nutritional stimuli: further support for the need for a big picture approach to tackling obesity and chronic disease. Obes Rev 2010;11:137149.
15 Hevener AL, Febbraio MA; Stock Conference Working Group: The 2009 stock conference report: inflammation, obesity and metabolic disease. Obes Rev 2010;11:635-644.

16 Cao Y: Adipose tissue angiogenesis as a therapeutic target for obesity and metabolic diseases. Nat Rev Drug Discov 2010;9:107-115.

17 Scaglione R, Di Chiara T, Cariello T, Lietata G: Visceral obesity and metabolic syndrome: two faces of the same medal? Intern Emerg Med 2010;5:111-119.

18 Nieuwenhuizen AG, Rutters F: The hypothalamic-pituitary-adrenal-axis in the regulation of energy balance. Physiol Behav 2008;94:16917.

19 Kleinridders A, Könner AC, Brüning JC: CNS-Targets in control of energy and glucose homeostasis. Curr Opin Pharmacol 2009;9:794-804.

20 Thaler JP, Choi SJ, Schwartz MW, Wisse BE: Hypothalamic inflammation and energy homeostasis: resolving the paradox. Front Neuroendocrinol 2010;31:79-84.

21 Hotamisligil GS: Inflammation and endoplasmic reticulum stress in obesity and diabetes. Int J Obes (Lond) 2008;32(suppl 7):S52-54.

22 Kyrou I, Tsigos C: Stress hormones: physiological stress and regulation of metabolism. Curr Opin Pharmacol 2009;9:787-793.

23 Halberg N, Wernstedt-Asterholm I, Scherer PE: The adipocyte as an endocrine cell. Endocrinol Metab Clin North Am 2008;37:753-768.

24 Baggio LL, Drucker DJ: Biology of incretins: GLP-1 and GIP. Gastroenterology 2007;132: 2131-2157.

25 Chen CY, Asakawa A, Fujimiya M, Lee SD, Inui A: Ghrelin gene products and the regulation of food intake and gut motility. Pharmacol Rev 2009;61:430-481.

26 Andersson CX, Gustafson B, Hammarstedt A, Hedjazifar S, Smith U: Inflamed adipose tissue, insulin resistance and vascular injury. Diabetes Metab Res Rev 2008;24:595-603.

27 Jordan SD, Könner AC, Brüning JC: Sensing the fuels: glucose and lipid signaling in the CNS controlling energy homeostasis. Cell Mol Life Sci 2010;67:3255-3273.

28 Sandoval D, Cota D, Seeley RJ: The integrative role of CNS fuel-sensing mechanisms in energy balance and glucose regulation. Annu Rev Physiol 2008;70:513-535.
29 Thaler JP, Choi SJ, Schwartz MW, Wisse BE: Hypothalamic inflammation and energy homeostasis: resolving the paradox. Front Neuroendocrinol 2010;31:79-84.

30 Straub RH, Kalden JR: Stress of different types increases the proinflammatory load in rheumatoid arthritis. Arthritis Res Ther 2009;11:114.

31 Libby P, Okamoto Y, Rocha VZ, Folco E: Inflammation in atheriosclerosis: transition from theory to practice. Circ J 2010;74:213220.

32 Löffler G, Petrides PE: Koordinierung des Stoffwechsels; in Löffler G, Petrides PE (eds): Biochemie und Pathobiochemie, ed 7. Berlin, Springer, 2003, pp 557-578.

33 Bazuine M, Stenkula KG, Cam M, Arroyo M, Cushman SW: Guardian of corpulence: a hypothesis on p53 signaling in the fat cell. Clin Lipidol 2009;4:231-243.

34 Fischer-Posovsky P, Wabitsch M: Entwicklung und Funktion des Fettgewebes. Monatsschr Kinderheilkd 2004;152:834-842.

35 de Ferranti S, Mozaffarian D: The perfect storm: obesity, adipocyte dysfunction, and metabolic consequences. Clin Chem 2008; 54:945-955.

36 www.who.int/mediacentre/factsheets/fs311/en.

37 Lionetti L, Mollica MP, Lombardi A, Cavaliere G, Gifuni G, Barletta A: From chronic overnutrition to insulin resistance: the role of fat-storing capacity and inflammation. Nutr Metab Cardiovasc Dis 2009;19:146-152.

38 Lee YS, Choi JW, Hwang I, Lee JW, Lee JH, Kim AY, Huh JY, Koh YJ, Koh GY, Son HJ, Masuzaki H, Hotta K, Alfadda AA, Kim JB: Adipocytokine orosomucoid integrates inflammatory and metabolic signals to preserve energy homeostasis by resolving immoderate inflammation. J Biol Chem 2010;285:2217422185.

39 Wood IS, de Heredia FP, Wang B, Trayhurn P: Cellular hypoxia and adipose tissue dysfunction in obesity. Proc Nutr Soc 2009;68:370377.

40 Mills CD, Kincaid K, Alt JM, Heilman MJ, Hill AM: M-1/M-2 macrophages and the Th1/ Th2 paradigm. J Immunol 2000;164:61666173.

41 Lumeng CN, Bodzin JL, Saltiel AR: Obesity induces a phenotypic switch in adipose tissue macrophage polarization. J Clin Invest 2007;117:175-184. 